\title{
An optimised linear mechanical model for estimating brain shift caused by meningioma tumours
}

\author{
Hossein Yousefi ${ }^{1}$, Alireza Ahmadian ${ }^{1, *}$, Davood Khodadad ${ }^{2}$, Hooshangh Saberi $^{3}$, \\ Alireza Daneshmehr ${ }^{4}$ \\ ${ }^{1}$ Department of Medical Physics and Biomedical Engineering, Tehran University of Medical Sciences (TUMS), \\ Research Centre for Biomedical Technology and Robotics, RCBTR, Tehran, Iran \\ ${ }^{2}$ Experimental Mechanics, Luleå University of Technology, SE-971 87 Luleå, Sweden, Exceptional Talents Development Centre, \\ Tehran, Iran \\ ${ }^{3}$ Department of Neurosurgery, Tehran University of Medical Sciences (TUMS), Brain and Spinal Injuries Repair Research Centre, \\ Tehran, Iran \\ ${ }^{4}$ Department of Mechanical Engineering, University of Tehran, Iran
}

\section{Email address:}

hossein_yousefy@yahoo.com(H. Yousefi), ahmadian@sina.tums.ac.ir(A. Ahmadian),dkhodadad@razi.tums.ac.ir(D. Khodadad), saberih@sina.tums.ac.ir(H. Saberi), daneshmehr@ut.ac.ir(A. Daneshmehr)

\section{To cite this article:}

Hossein Yousefi, Alireza Ahmadian, Davood Khodadad, Hooshangh Saberi, Alireza Daneshmehr. An Optimised Linear Mechanical Model for Estimating Brain Shift Caused by Meningioma Tumours. International Journal of Biomedical Science and Engineering. Vol. 1, No. 1, 2013, pp. 1-9. doi: 10.11648/j.ijbse.20130101.11

\begin{abstract}
Estimation of brain deformation plays an important role in computer-aided therapy and image-guided neurosurgery systems. Tumour growth can cause brain deformation and change stress distribution in the brain. Biomechanical models exist that use a finite element method to estimate brain shift caused by tumour growth. Such models can be categorised as linear and non-linear models, both of which assume finite deformation of the brain after tumour growth. Linear models are easy to implement and fast enough to for applications such as IGS where the time is a great of concern. However their accuracy highly dependent on the parameters of the models in this paper, we proposed an optimisation approach to improve a naive linear model to achieve more precise estimation of brain displacements caused by tumour growth. The optimisation process has improved the accuracy of the model by adapting the brain model parameters according to different tomour sizes.We used patient-based tetrahedron finite element mesh with proper material properties for brain tissue and appropriate boundary conditions in the tumour region. Anatomical landmarks were determined by an expert and were divided into two different sets for evaluation and optimisation. Tetrahedral finite element meshes were used and the model parameters were optimised by minimising the mean square distance between the predicted locations of the anatomical landmarks derived from Brain Atlas images and their actual locations on the tumour images. Our results demonstrate great improvement in the accuracy of an optimised linear mechanical model that achieved an accuracy rate of approximately $92 \%$.
\end{abstract}

Keywords: Brain Deformation, Finite Element Modelling, Linear and Non-Linear Brain Models, Brain Tumour, Tumour Growth

\section{Introduction}

Important achievements in engineering have been achieved by mathematical modelling and computer simulation of brain shift. Soft tissue deformation modelling has received increasing attention in the biomedical imaging community. The surgical simulation research goals are to model and simulate deformable tissues for applications requiring real-time interaction. Medical applications for modelling and simulation include simulation-based training, skills assessment and operation planning[1] Image guided intervention systems can help surgeons improve the clinical outcomes of surgery. Modelling and simulation are particularly important in certain areas, such as tumour growth, oedema, hematomas and craniotomy motion 
tracking and segmentation. However, soft tissue simulations are often plagued by imprecise geometric information, unknown constitutive laws, boundary conditions and distributed forces[2].

In this paper, we used 3D modelling with the finite element method to elucidate the effect of brain tumour mass. Several approaches have been developed to address brain deformation. Recently, biomechanical models have been developed that estimate displacements. These models are based on physical brain deformation and thus require measurements after deformation. Tissue deformation simulation usually starts with segmentation of the target geometry from a medical image, which is then used to reconstruct a representation of the target geometry's boundary surface. Some models can model brain responses to strain and stress. Some such models are linear and assume that the stress and strain relationship is linear, while others assume a non-linear relationship. Linear models assume that the brain's response to stress and strain is similar to that of elastic or solid materials[3]. A study by Hamidian et al. proved that the solid mechanical model is more reliable than the elastic model. However, more recent research has indicated that the brain's response to stress and strain is non-linear, which suggests that the brain is best described as a viscoelastic material. The linear models have low computational complexity, and easy to implement but relatively more estimation error than non-linear models, which are more complex, and time consuming.

Hogea et al. used a linear elastic model to simulate brain behaviour in combination with a tumour model to model brain deformation. They evaluated a linear model for two dog cases and one human case and found that in the human case the model error was high[4]. In other studies, purely mechanical models were used to simulate evolution of the tumour-brain interface and tumour mass effect[5-7]. In these cases, the brain tissue was modelled as a non-linear elastic material, a cavity representing the tumour was introduced and a pressure-like Neumann condition was used to model tumour-induced interface forces. Ashraf et al. used a non-linear model to simulate tumour mass effect and modelled glioma tumour mass effect and oedema and applied it for the registration method[7]. Their results showed that using the mass effect of the brain tumour model combined with the registration method can improve registration results.

To estimate brain deformation caused by tumours, we used a brain mechanical model combined with a tumour growth model. The main motivation of this study was to optimise a model for brain tumour segmentation and registration. In previous studies, the deformation force was obtained from pre-registration of normal data and deformed data[8, 9]. Because we used tumour growth to compute external forces that affect the brain model, our model is more reliable than others.

Computer Aided Therapy (CAT) requires a better understanding of the characteristics of brain cancer progression based on phenotypic cancer profiles derived from imaging, histopathology and other sources, which can ultimately help determine predictive factors of cancer invasiveness. Significant tools for understanding such cancer profiles are statistical atlases. In brain tumour patients, such atlases have the potential to assist surgical and treatment planning[10].

The decision of whether and how to best treat a brain tumour is based on multiple factors, including size and location of the tumour, tumour type, symptoms, growth rate and age of the patient (among others). In general, there are two basic options: surgical removal and radiation. In both cases (surgical and radiation) segmentation has an impressive impact on Image Guided Surgery (IGS) and CAT systems. Brain MRIs that reveal tumours are difficult to segment because of brain tissue deformation caused by tumour mass effect or volume expansion[11].

To model and estimate deformation of brain structure, we focused on one type of brain tumour, meningiomas, because they are a good representative of brain tumours in general and possess several attractive characteristics. Meningiomas arise from a layer of tissue (the meninges) that covers the brain and spine. Meningiomas grow on the surface of the brain (or spinal cord) and therefore push the brain away rather than growing from within. Meningiomas represent about 25 percent of all tumours originating in the head. Meningiomas are often slow growing, increasing in size only 1-2 mm per year, there for we can assume that the tomour growth rate is 1-2 $\mathrm{mm}$ per year, and their growth behaviour can most closely be described as linear, so we can assume that their growth rate is linear. Finally, meningiomas have sharp margins and rarely invade neighbouring tissue[12].

In sum, our proposed method can properly estimate brain displacement because of the optimised model parameters and external force calculation by the tumour growth model.

\section{Materials \& Methods}

\subsection{Pre-Processing}

We have difficulties in finding suitable cases with meningioma tumour at least two stage of tumour growth but we managed to find seven cases with different variation in tumour size and location which could validate our results statically. Patient geometric data used in this paper were obtained from a set of seven MRIs of patients with brain meningioma tumours obtained from the Imaging Centre of Imam Khomeini Hospital and surgical planning laboratory of Harvard University. These data have a resolution of $256 \times 256 \times 124$ with pixel size of $0.9375 \times 0.9375 \mathrm{~mm}$ and slice thickness of $1.5 \mathrm{~mm}[13,14]$. Atlas data was obtained from brain-web with resolution of $256 \times 256 \times 100$ pixels[21]. Type and location of tumours are shown in Table 1.

Because MRI images only elucidate tumoural brain anatomy, the ability to predict soft tissue deformation, and therefore tumoural brain anatomy during tumour growth, is a primary requirement for reliable treatment. If 
displacements within the brain can be computed, they can be used to simulate tumours in healthy atlases. A block diagram of the method presented in this paper is sketched in Figure 1.

Table 1. Type and location of brain tumors in patient data that obtained from imaging center of Imam Khomeini hospital and surgical planning laboratory of Harvard University.

\begin{tabular}{cccccccc}
\hline & Case 1 & Case 2 & Case 3 & Case 4 & Case 5 & Case 6 & Case 7 \\
\hline Type of tumor & Menengioma & Menengioma & Menengioma & Menengioma & Menengioma & Menengioma & Meningioma \\
Location of tumor & left frontal & right parietal & right parietal & right frontal & right parietal & Left occipital & left frontotemporal \\
\hline
\end{tabular}

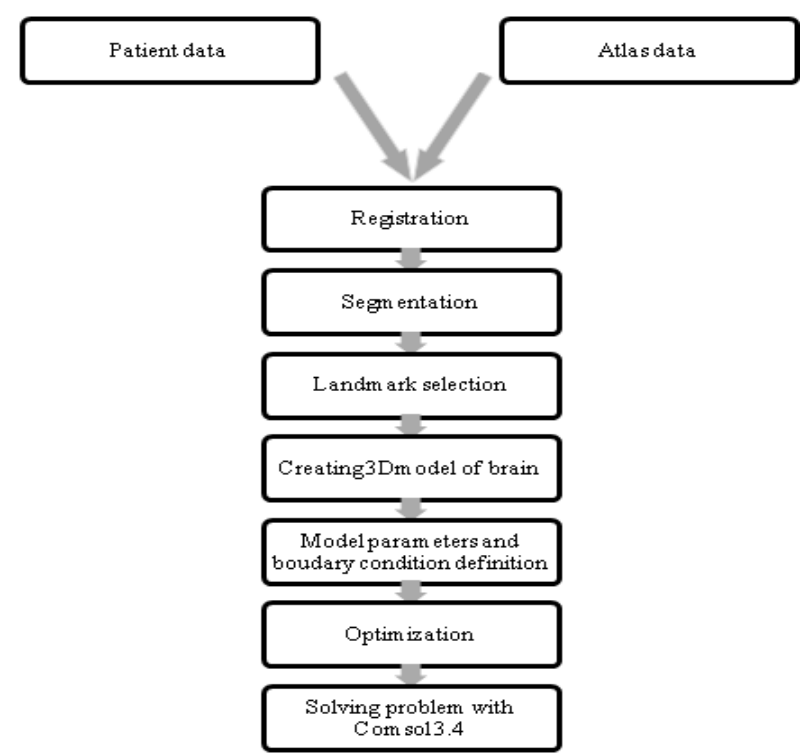

Figure 1. block diagram of proposed method for estimation of brain deformation

The atlas data were registered non-rigidly with patient data to reduce misalignment errors. The non-rigid B-spline method was used to register atlas data to the patient dataset, as detailed in Equation 1. Figure 2 shows the results of the registration process for cases 2, 3 and 4, which had tumours in the right parietal, right parietal and right frontal lobes, respectively.

$$
\begin{aligned}
& D(x)=\sum_{i=0}^{3} \sum_{j=}^{3} \sum_{k=0}^{3} B_{l}(u) B_{m}(v) B_{n}(w) P_{i+l, j+m, k+n} \\
& l=\left[x / n_{x}\right]-1 \quad m=\left[y / n_{y}\right]-1 \quad n=\left[z / n_{z}\right]-1 \\
& B_{0}(t)=\left(-t^{3}+3 t^{2}-3 t+1\right) / 6 \\
& B_{1}(t)=\left(3 t^{3}-6 t^{2}+4\right) / 6 \\
& B_{2}(t)=\left(-3 t^{3}+3 t^{2}+3 t+1\right) / 6 \\
& B_{3}(t)=t^{3} / 6
\end{aligned}
$$

Where $\mathrm{i}=\left[\mathrm{x} / \mathrm{n}_{\mathrm{x}}\right]-1, \mathrm{j}=\left[\mathrm{y} / \mathrm{n}_{\mathrm{y}}\right]-1$, and $\mathrm{k}=\left[\mathrm{z} / \mathrm{n}_{\mathrm{z}}\right]-1$, denote the index of the CP cell containing ( $x, y, z)$ and $\mathrm{u}, \mathrm{v}$ and $\mathrm{w}$, which are relative positions of $(x, y, z)$ in three dimensions. $\mathrm{B}_{0}$ through $\mathrm{B}_{3}$ are cubic B-splines[20].

In order to specify brain tissue displacement, anatomical landmarks are defined in both patient data and registered image data as shown in Figure 3. Landmarks, such as ventricle borders and the brain midline, are chosen close to tumour regions so that deformation can be effectively tracked as the tumour shifts these landmarks.
In order to better distinguish between the brain parenchyma, ventricles and tumours when creating 3D brain models, the images were first segmented manually in two areas as shown in Figure 4 using open source 3D SLICER software[22].
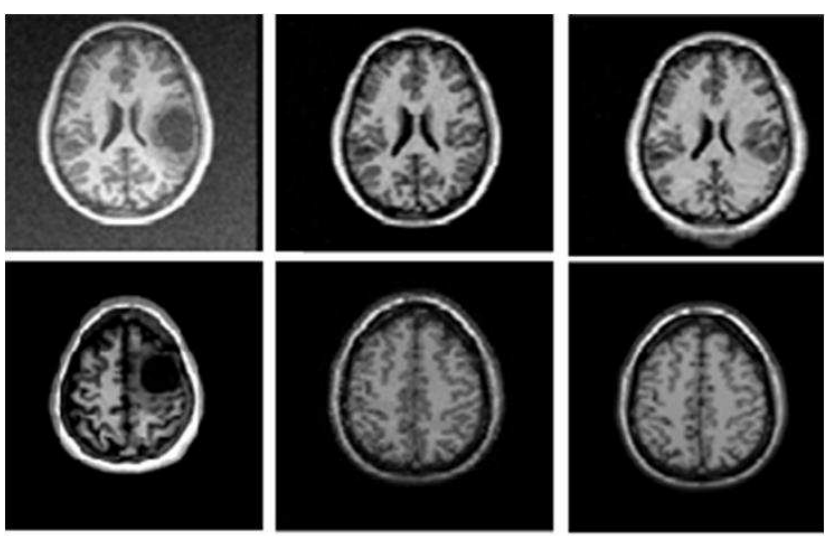

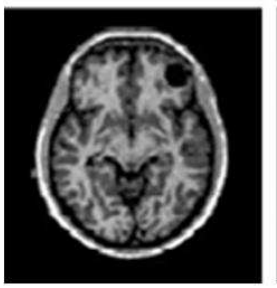

Column (a)

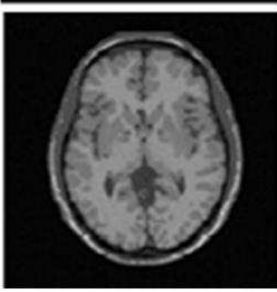

column (b)

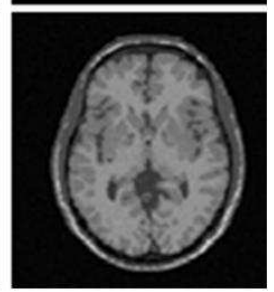

column (c)
Figure 2. Registration of atlas data with patient data (case 2, 3, 4) left column patient data middle atlas data right column registered data. In this study we used the registered image to build a computational model of the brain, and patient image to measure the displacement of the exposed surface of the brain (used as loading of the model) and to validate computational results. Surfaces were determined from the images provided by Department of Surgery, Imam Khomeini Hospital Tehran University of Medical Science, Iran and Brigham and Women's Hospital (Harvard Medical School, Boston, Massachusetts, USA)
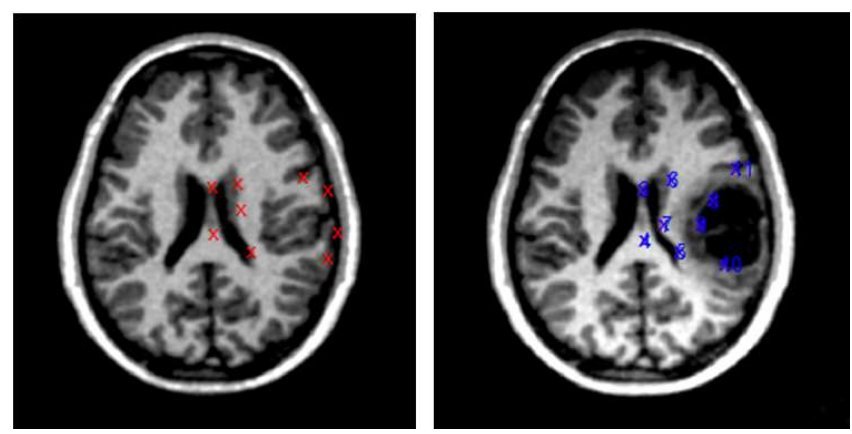

Figure 3. selecting landmarks in atlas and tumoural image. These landmarks are selected manually by experts in the regions that we can track the deformation of brain tissue 

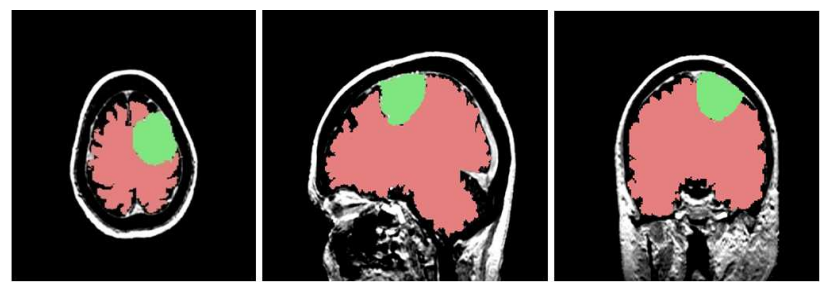

Figure 4. Segmented MRIs of the patient head used for building patient specific brain mesh. The tumor segmentation is indicated by whit green color.

After segmentation, 3D models of surfaces of the brain parenchyma and tumours were created as shown in Figure 5.

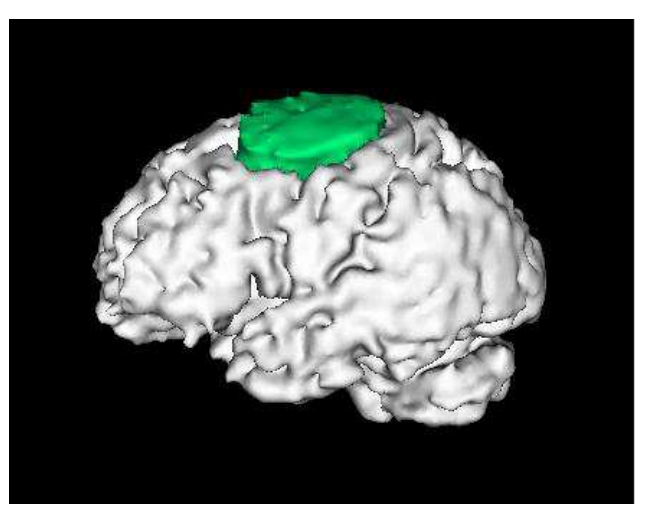

Figure 5. 3D model of the brain parenchyma, and tumor surface which was applied to build the patient specific brain mesh

A necessary step in obtaining the numerical model of the brain is the creation of a computational grid, which in most practical grids is a finite element mesh. Because of the long computation time requirements, meshes with low-order elements must be constructed that are not computationally costly. Many algorithms are available for fast and accurate automatic mesh generation using tetrahedral elements[2]. For obtaining the accurate results we choose the tumour seeds manually in regions that tumour exists in the real data and grow up there. In the case of severe pathologies (such as a brain tumour), many authors proposed the use of tetrahedral meshes for their models, and therefore, tetrahedral meshes were used here for model creation as shown in Figure 6.

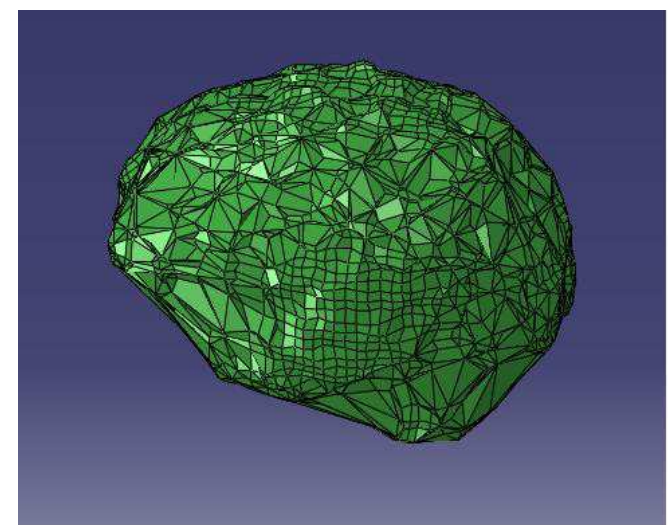

Figure 6. tetrahedron-dominant brain mesh constructed in this study

\subsection{Mechanical Properties of Brain Tissue}

\subsubsection{Linear Model}

A linear model of the brain was used to determine brain deformation. It has been shown that such models provide numerical formulations that sufficiently describe brain tissue behaviour[3]. In addition, linear models are simpler to implement and run relatively fast. A linear model was used here because the brain is assumed to be a linear continuum with no initial stresses or strains. The energy of the brain's deformation caused by externally applied forces can be expressed as Equation 2:

$$
W=\int_{\Omega} \sigma^{T} \varepsilon d \Omega+\int_{\Omega} F^{T} u d \Omega
$$

where $\mathrm{F}=\mathrm{F}(\mathrm{x}, \mathrm{y}, \mathrm{z})$ is the total force applied to the brain, external force obtained from $\Omega$ is the brain, $u$ is the displacement vector, and $\square$ is the strain vector that can be defined as Equation 3:

$$
\varepsilon=\left(\frac{\partial u}{\partial x}, \frac{\partial u}{\partial y}, \frac{\partial u}{\partial z}, \frac{\partial u}{\partial x}+\frac{\partial u}{\partial y}, \frac{\partial u}{\partial y}+\frac{\partial u}{\partial z}, \frac{\partial u}{\partial x}+\frac{\partial u}{\partial z}\right)
$$

In addition, $\sigma$ is the stress vector, and in the case of linear elasticity with no initial stresses or strains it relates to the strain vector by the linear equation $\sigma=\mathrm{D} \square$, where $\mathrm{D}$ is the elasticity matrix describing the material properties and is described in Equation 4:

$$
D=\frac{E(1-v)}{(1+v)(1-2 v)}\left[\begin{array}{cccccc}
1 & \frac{v}{1-v} & \frac{v}{1-v} & 0 & 0 & 0 \\
\frac{v}{1-v} & 1 & \frac{v}{1-v} & 0 & 0 & 0 \\
\frac{v}{1-v} & \frac{v}{1-v} & 1 & 0 & 0 & 0 \\
0 & 0 & 0 & \frac{1-2 v}{2(1-v)} & 0 & 0 \\
0 & 0 & 0 & 0 & \frac{1-2 v}{2(1-v)} & 0 \\
0 & 0 & 0 & 0 & 0 & \frac{1-2 v}{2(1-v)}
\end{array}\right]
$$

The value of $\mathrm{D}$ is obtained by two material parameters: Young's modulus (E), which relates tension and stretch in the main orthogonal directions, and the Poisson ratios (v), which represent the ratio of the lateral contraction due to longitudinal stress in a given plane. Deformation of the brain is determined by solving Equation 1 for the displacement vector $u$, which minimises the energy function E. The Numerical solution to these equations could be written as the global linear Equation 5:

$$
K u=-F
$$

The solution of Equation 4 defines the deformation field that results from the forces applied to the brain. External force is obtained from tumour growth. Initial coefficients (Young's modulus $=3000 \mathrm{~Pa}$, Poisson ratio $=0.45$ ) were chosen as previously described by Ferrant et al.[15]. 


\subsubsection{Optimised Linear Model}

The brain model parameters, called Poisson ratio and Young's modulus in the linear method, can be influenced by the patient's brain anatomy and the type of tumours being modelled. Tumour sizes and brain anatomy can change the above parameters from patient to patient and for different types of tumours. Therefore, to obtain the best model parameters and to achieve accurate results, an optimisation process was utilised in combination with the conventional linear model to obtain the best parameters for each patient. In this study, meningioma tumours from 7 patients were analysed. The cost function was defined as the mean square difference of distance between the landmark position manually selected in the real image data and their corresponding estimated positions in the model as explained in Equation 6. Landmarks were selected in a non-uniform manner around the tumour contour focusing on the areas with large deformations as shown in Figure 3. One half of the landmarks were used in the optimisation process and the other half were used for error calculation. The Matlab optimisation toolbox was used, which is based on a linear algorithm to optimise the following cost function:

$$
M S E=\frac{1}{M} \sum_{i=1}^{M}\left(x_{i E}-x_{i D}\right)^{2}
$$

Where $M$ is the number of landmarks, $X_{i E}$ is the estimated landmark position and $\mathrm{X}_{\mathrm{iD}}$ is the corresponding landmark position the in real data.

\subsubsection{Non-Linear Model}

As mentioned above, brain shift behaves in a non-linear manner and is modelled as a viscoelastic material. Nonlinear models closely approximate real shift, and in comparison with linear models they achieve more accurate results, but have higher computational costs. To model deformations induced by tumours more precisely, the Ogden-based Hyper-viscoelastic constitutive model was used in the following Equations 7 and 8[2, 15-17]:

$$
\begin{gathered}
W=\frac{2}{\alpha^{2}} \int_{0}^{t}\left[\mu(t-\tau) \frac{d}{d \tau}\left(\lambda_{1}^{\alpha}+\lambda_{2}^{\alpha}+\lambda_{3}^{\alpha}-3\right)\right] d \tau \\
\mu=\mu_{0}\left[1-\sum_{k=1}^{n} g_{k}\left(1-e^{\frac{-t}{\tau_{k}}}\right)\right]
\end{gathered}
$$

Where $\mathrm{W}$ is the strain energy. $\lambda 1, \lambda 2, \lambda 3$ (directions 1,2 , 3 corresponding to $\mathrm{x}, \mathrm{y}, \mathrm{z}$ ) are principal extensions and their values are 1 for no deformation, greater than 1 for extension and smaller than 1 for compression. $\alpha$ is a material coefficient without physical meaning. The value of $\alpha$ was found to be -4.7 , see Table $2[2,16]$, $t$ and $\tau$ denote time. Equation 8 describes viscous response of the tissue. $\mu 0$ is the instantaneous shear modulus in the un-deformed state. $\tau_{\mathrm{k}}$ are characteristic relaxation times. Stress-strain relationships are obtained by differentiating the energy function $\mathrm{W}$ with respect to strains[2, 16].

Table 2. Nonlinear brain tissue model parameters for Eqs (5) and (6)

\begin{tabular}{ccc}
\hline Instantaneous response & $\mathbf{k}=\mathbf{1}$ & $\mathbf{k}=\mathbf{2}$ \\
\hline$\mu 0=842[\mathrm{~Pa}]$ & characteristic time & characteristic time \\
$\alpha=-4.7$ & $\mathrm{t} 1=.5[\mathrm{~s}]$ & $\mathrm{t} 2=50[\mathrm{~s}]$ \\
& $\mathrm{g} 1=0.450$ & $\mathrm{~g} 2=0.365$ \\
\hline
\end{tabular}

The stress-strain rate relationships are non-linear, and the stiffness of the brain in compression is much higher than in extension.

To compute the external force, the tumour growth rate was used. An interesting consequence of the basic model assumptions is that the profile of the concentration of tumour cells depends on the ratio of the growth rate Equation 9[7, 18]:

$$
\frac{\partial c}{\partial t}=\frac{\rho\left(c_{s}-c\right)}{c_{s}}
$$

In which $\mathrm{c}(\mathrm{x}, \mathrm{t})$ designates the tumour cell density at location $\mathrm{x}$ and time $\mathrm{t}$ and $\rho$ denotes the net proliferation rate. This computation makes our method more accurate due to proper force calculation for each patient. Therefore, brain displacement due to tumour growth can be measured by this method. Displacement by tumour is defined as a homogenous force applied to the brain that must satisfy the following condition[19]:

$$
f(e x t)+\operatorname{div}(\sigma-\lambda I c(t))=0
$$

Where $\mathrm{f}(\mathrm{ext})$ is external force, $\sigma$ is internal stress, $\mathrm{c}(\mathrm{t})$ is tumour growth model and $\lambda$ is coupling factor. A coupling factor was chosen that minimises the quantitative difference between the model and the real deformations: $1.4 \mathrm{~N} \mathrm{~mm} / \mathrm{Cells}[8]$. This equation is the differential version of the law proposed by Wasserman[19] and can be locally interpreted as a tissue internal pressure proportional to the tumour concentration.

\section{Results}

Biomechanical simulation of brain tumour shift took approximately 56.50 minutes and 18.00 minutes of computation time on a personal computer with a $2.53 \mathrm{GHz}$ Pentium processor with $4 \mathrm{~GB}$ of Ram to predict brain deformation using a non-linear and linear model, respectively. Non-rigid B-spline registration was used to align atlas data to patient data. Then, segmentation was applied to our dataset to extract brain tissue from cranial bone. Finally, the 3D model of the brain based on tetrahedron finite element mesh was created as shown in Figure 6. We used the COMSOL3.4 software to implement the model, which is based on the finite element method for solving partial differential equations. To evaluate the efficiency of the created model in relation to real data, we 
used images of seven patients with meningioma tumours and normal brain atlas MRIs.

Table 3. Here, Atlas and tumoral MR images of seven patients with brain tumor (meningioma) are used. The atlas and patient images are registered none rigidly, and then pairs of anatomical landmarks are determined by radiologist in the corresponding images. Based on the error of the landmarks, ability of linear model to estimate real brain deformations by tumor is evaluated.

\begin{tabular}{lccccccc}
\hline & case 1 & case 2 & case 3 & case 4 & case 5 & case 6 & case 7 \\
\hline Max error in $\mathrm{x}(\mathrm{mm})$ & 9.71 & 9.32 & 7.34 & 7.91 & 7.25 & 10.14 & 6.73 \\
Max error in $\mathrm{y}(\mathrm{mm})$ & 8.62 & 8.52 & 7.52 & 7.78 & 6.79 & 9.91 & 5.94 \\
Max error in $\mathrm{z}(\mathrm{mm})$ & 7.87 & 6.51 & 5.15 & 5.31 & 4.87 & 7.53 & 3.15 \\
Min error in $\mathrm{x}(\mathrm{mm})$ & 3.8 & 3.9 & 3.28 & 2.54 & 2.22 & 4.16 & 2.35 \\
Min error in $\mathrm{y}(\mathrm{mm})$ & 2.95 & 3.2 & 2.91 & 3.21 & 2.15 & 3.85 & 2.15 \\
Min error in $\mathrm{z}(\mathrm{mm})$ & 2.55 & 2.75 & 2.25 & 2.16 & 1.85 & 2.85 & 2.05 \\
Mean error in $\mathrm{x}(\mathrm{mm})$ & 7.95 & 7.15 & 5.15 & 5.25 & 5.78 & 7.76 & 4.95 \\
Mean error in $\mathrm{y}(\mathrm{mm})$ & 6.35 & 6.37 & 5.38 & 5.23 & 4.92 & 7.75 & 4.25 \\
Mean error in $\mathrm{z}(\mathrm{mm})$ & 5.65 & 5.24 & 4.15 & 4.15 & 3.15 & 6.22 & 2.75 \\
Error percent & $15.65 \%$ & $18.00 \%$ & $17.50 \%$ & $18.36 \%$ & $16.80 \%$ & $19.20 \%$ & $17.15 \%$ \\
\hline
\end{tabular}

Table 4. Here, Atlas and tumoral MR images of seven patients with brain tumor (meningioma) are used. The atlas and patient images are registered none rigidly, and then pairs of anatomical landmarks are determined by radiologist in the corresponding images. These landmarks used for the testing the model validation. Based on the error of the landmarks, ability of optimized linear model to estimate real brain deformations by tumor is evaluated

\begin{tabular}{|c|c|c|c|c|c|c|c|}
\hline & case 1 & case 2 & case 3 & case 4 & case 5 & case 6 & case 7 \\
\hline Max error in $\mathrm{x}(\mathrm{mm})$ & 4.72 & 4.31 & 3.87 & 4.91 & 3.25 & 5.15 & 3.71 \\
\hline Max error in $y(m m)$ & 4.54 & 4.66 & 4.26 & 4.84 & 3.83 & 5.89 & 2.93 \\
\hline Max error in $\mathrm{z}(\mathrm{mm})$ & 1.88 & 2.18 & 26 & 2.32 & 1.73 & 2.5 & 1.91 \\
\hline Mean error in $\mathrm{x}(\mathrm{mm})$ & 1.87 & 1.63 & 1.38 & 1.55 & 1.14 & 2.17 & 1.53 \\
\hline Mean error in $\mathrm{y}(\mathrm{mm})$ & 1.54 & 1.28 & 1.89 & 1.96 & 1.42 & 2.31 & 1.38 \\
\hline Mean error in $\mathrm{z}(\mathrm{mm})$ & 0.83 & 0.97 & 0.71 & 0.62 & 0.55 & 1.41 & 0.99 \\
\hline Error percent & $7.27 \%$ & $7.58 \%$ & $6.91 \%$ & $6.70 \%$ & $5.40 \%$ & $8.10 \%$ & $7.82 \%$ \\
\hline
\end{tabular}

Table 5 Here, Atlas and tumoral MR images of seven patients with brain tumor (meningioma) are used. The atlas and patient images are registered none rigidly, and then pairs of anatomical landmarks are determined by radiologist in the corresponding images. These landmarks used for the testing the model validation. Based on the error of the landmarks, ability of nonlinear model to estimate real brain deformations by tumor is evaluated.

\begin{tabular}{|c|c|c|c|c|c|c|c|}
\hline & case 1 & case 2 & case 3 & case 4 & case 5 & case 6 & case 7 \\
\hline Max error in $\mathrm{x}(\mathrm{mm})$ & 3.92 & 3.75 & 3.37 & 4.28 & 2.81 & 4.52 & 3.21 \\
\hline Max error in $\mathrm{y}(\mathrm{mm})$ & 4.15 & 3.92 & 4.17 & 4.31 & 3.29 & 5.41 & 2.45 \\
\hline Max error in $\mathrm{z}(\mathrm{mm})$ & 1.58 & 1.85 & 1.67 & 2.03 & 1.34 & 2.13 & 1.52 \\
\hline Mean error in $\mathrm{x}(\mathrm{mm})$ & 1.21 & 1.34 & 1.17 & 1.36 & 0.91 & 1.82 & 1.24 \\
\hline Mean error in $y(\mathrm{~mm})$ & 1.09 & 0.81 & 1.35 & 1.52 & 1.09 & 2.12 & 1.03 \\
\hline Mean error in $\mathrm{z}(\mathrm{mm})$ & 0.63 & 0.77 & 0.62 & 0.55 & 0.45 & 1.13 & 0.79 \\
\hline Error percent & $6.35 \%$ & $6.65 \%$ & $6.15 \%$ & $5.9 \%$ & $4.5 \%$ & $6.95 \%$ & $6.77 \%$ \\
\hline
\end{tabular}

The displacement vector of brain tissue caused by tumours was obtained calculated by the model. The improved agreement between the computed and observed displacements has enhanced the model's ability to predict deformation. As can be observed visually, the tumour simulated by this model resembles the real tumour imaged in a patient. Figure 7 shows the axial, coronal and sagittal views of the real brain tumour and the corresponding predicted model. Comparison of real and modelled tumours shows a good matchThe red to yellow color of estimated 
deformation field can present the tomour region and the green color presents the regions of brain that deformation due to tumour exist.

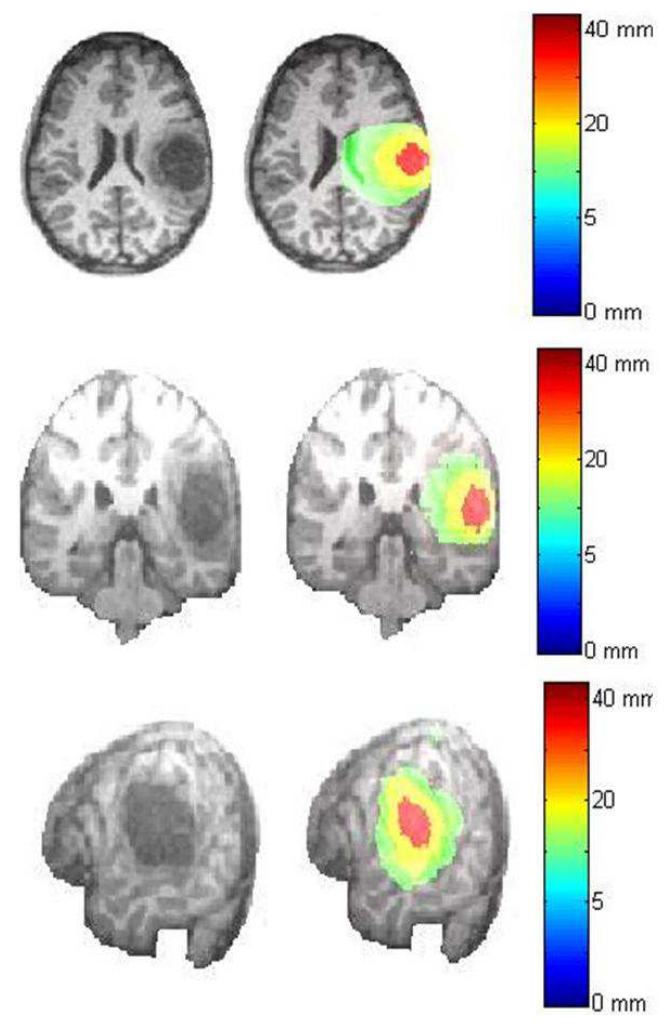

Figure 7. Real brain tumor (left column) and simulated one (right column) with optimized linear mechanical model: upper row axial axis, middle row coronal axis and lower row sagital axis case 3. This patient has a meningioma in right parietal lobe of his brain. The red to yellow color of estimated deformation field can present the tomour region and the green color presents the regions of brain that deformation due to tomour exist.

To quantify the accuracy of the simulation, a medical expert manually selected corresponding feature points on the patient MRIs. To estimate these landmark displacements, the measured displacements could then be compared to the ones shifted by the models. Detailed comparison of the cross sections of actual tumour shift and tumour shift predicted by the three brain models is presented in Tables 3, 4 and 5 and Figures 8, 9 and 10.

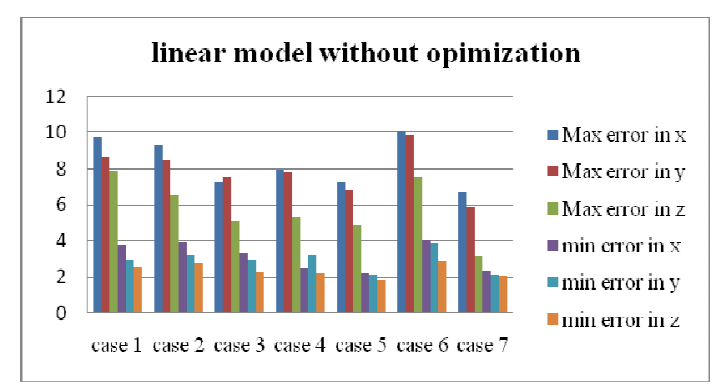

Figure (8) error comparison of seven cases in linear model without optimization

After optimisation of model parameters, the accuracy of the linear solid mechanical model increased. As showed in
Table 4 and Figure 9, the model accuracy increased by about $15 \%$.

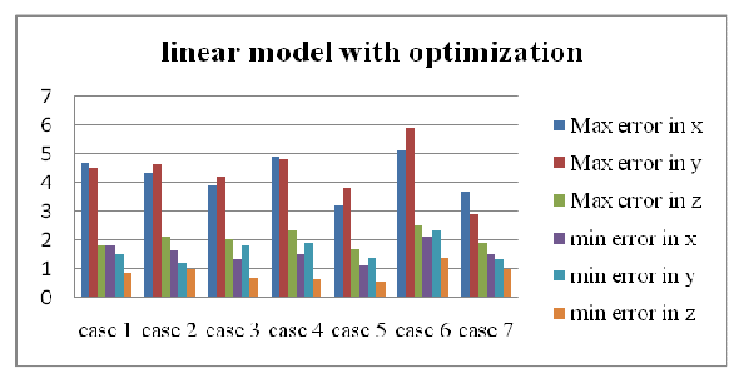

Figure 9. error comparison of seven cases in linear model with optimization

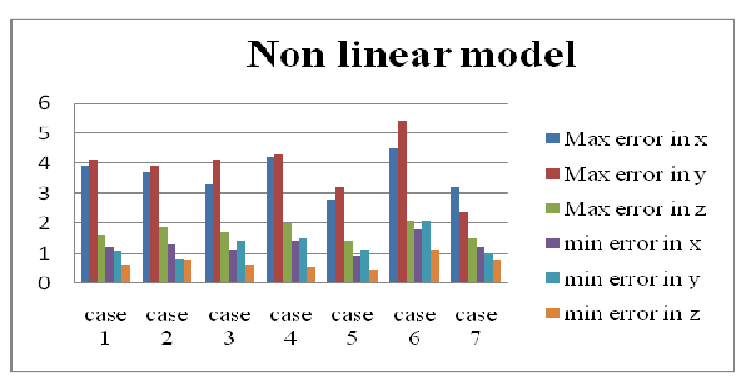

Figure 10. error comparison of seven cases in nonlinear model

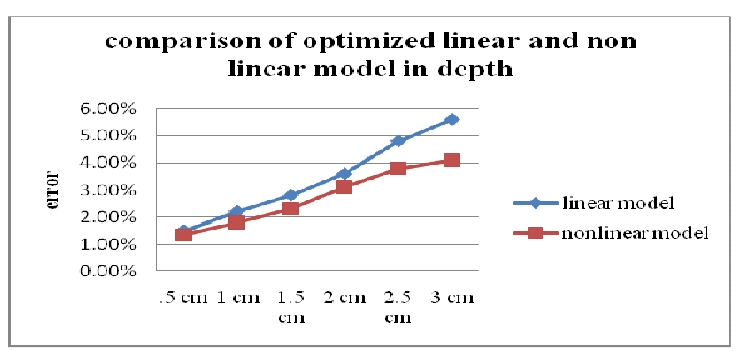

Figure 11. comparison of linear and nonlinear model as depth increase the nonlinear model has better response in contrast of linear model. Because nonlinear model considered the viscose property of brain but in linear model there is not any parameter that controls the viscose property.

In order to evaluate the two model types, we compared them in different depths of the brain. Our findings showed that, as we moved from the surface of the brain to deeper parts, the non-linear model achieved better results than the linear model, as depicted in Figure 11. This is likely because the non-linear model takes into account the viscose nature of the brain while the linear model does not. Thus, in cases with large deformations in which the viscose character of the brain changes as a result of tumour growth load, the non-linear model is recommended. It must be noted that implementing a linear solid mechanical model takes one-third times less time than implementing a nonlinear model. For example, the speed of implementation for the non-linear model and the linear mechanical model were 56.50 minutes and 18.00 minutes, respectively. In our study, the accuracy of the optimised linear mechanical model was similar to the non-linear model for displacement of up to $2.5 \mathrm{~cm}$ as shown in Figure 11. Therefore, the linear solid mechanical model is more appropriate and is highly 
recommended for calculation of deformations because of its speed and acceptable accuracy.

Table 6. Initial and optimized parameters value for linear model. These parameters change from patient to patient because of brain anatomy and tumor size

\begin{tabular}{lcc}
\hline & Initial value & Optimized value \\
\hline Young modules $(\mathrm{kp})$ & 3000 & $3000 \pm 250$ \\
Poisson ratio & .45 & $.45 \pm .145$ \\
\hline
\end{tabular}

The tolerance of the model parameters and the error depended on tumour size, position and depth, and the position of the selected landmarks also affects the results. Based on our findings, we propose that landmarks be selected in places where large displacements have occurred Therefore, we selected landmarks mostly around the tumour. This model is sensitive to Young's modulus and passion ratio, and thus these parameters should be optimised because patients have different brain anatomy and different types of tumours. The deviations of the model parameters after optimisation based on Equation 6 are presented in Table 6 .

\section{Discussion}

In this paper we focused on improving the well known linear model, which has been used expensively in previous works. Because of its simplicity, fastness and few model parameters we did not concentrate on non linear models which are already found very complex and time consuming with more model parameters to be chosen. However, in order to show how close an optimized linear model could be to a nonlinear model, we implemented a visicoelastic model to compare to our optimised linear model.

In estimation of brain deformation, model selection and optimisation of model parameters are important steps toward obtaining accurate and reliable results. Here, we computed the deformation of brain tissue resulting from meningiomas. We used tumour growth model to compute the external forces and we choose the tumour seeds manually in the regions that tumour exists in real data. It must be noted that because of limitations in obtaining patient data at different stages of tumour growth, we only considered the last stage in all cases. The external force on the brain increases with tomour growth and the brain tissue becomes dens. This will cause changing the brain parameters (Young's modulus and Poisson rate). If these parameters remain the same for all patients, the results of brain shift estimation due to different tumor size will not have sufficient accuracy. But when the brain tissue parameters were optimized for each patient as shown in Table 3 and 4 the results became closer to actual values. This improvement in the results of optimization process emphasizes the necessity of the optimization process in the linear model.
Finite element formulation combined with the tumour growth model resulted in very good agreement between the computed and measured displacements. As the results illustrated, the accuracy of displacement predicted by the optimised model is similar to the real data. Accuracy rates were $92 \%$ in the optimised linear model and $95 \%$ in the non-linear model but the computation time of the linear model is three times less than nonlinear model. Because of that the computation time is important in operating room, so our optimized model with less computation time and accuracy close to nonlinear model is proper to brain shift computation. Although there were areas with more displacement errors in the traditional linear model, our optimisation approach particularly in the deep brain parts, exhibited much better overall agreement between the predicted and real brain shifts. Visual inspection by experts also demonstrated this improved accuracy in the optimised linear solid mechanical model. We showed that the time required to implement an optimised linear model is less than a non-linear model by an order of one-third. As shown in tables 2, 3 and 4 the accuracy of the optimised linear mechanical model is almost the same as the nonlinear model. Therefore, the proposed optimised linear solid mechanical model is applicable for computation of brain deformations caused by meningiomas tumour because of its speed and acceptable accuracy. This method can be used in CAT systems to define the tumour and therapy volume more precisely. This will in turn improve therapy planning and reduce safe tissue radiation. Another application of this model is in image guided surgery (IGS). IGS systems, which involve simulation of the brain tumour and its location, allow surgical planning and less invasive procedures. Finally, this model can be used in combination with brain segmentation and registration algorithms to achieve higher accuracy rates.

The main limitation of this work is the relatively small number of patients with meningioma tumours from whom data were obtained and the lack of information about tumour staging. As mentioned earlier, brain parameters differ from patient to patient because of differences in brain anatomy and tumour size. In our approach, brain parameters were optimised for each patient. This optimisation can be further generalised to create a reference table of best model parameters for different patients. In this paper, we focused on meningioma tumours and did not consider the oedema effect of glioma tumours, but our method can be generalised to consider oedema effects by adding the diffusion term to the tumour growth model. We are currently working on evaluating the relevance of the model on more patient datasets and using this optimised model for deformable registration.

\section{Acknowledgments}

We thank Tehran University of Medical Sciences and the Research Center for Science and Technology in Medicine for supporting this project. We also thank Drs. Simon 
Warfield, Michael Kaus, Ron Kikinis, Peter Black and Ferenc Jolesz for sharing the tumour database of The Surgical Planning Laboratory[SPL], Department of Neurosurgery[NSG] and Department of Radiology of the Harvard Medical School at the Brigham and Women's Hospital, Boston, MA.

\section{References}

[1] Oden JT, Research directions in computational mechanics. Computer Methods in Applied Mechanics and Engineering 2003; 192: 913-22.

[2] Miller K, Wittek A, Joldes R, et al. Modelling brain deformations for computer-integrated neurosurgery. Communications In Numerical Methods In Engineering 2009. DOI: $10.1002 / \mathrm{cnm} .1260$

[3] Hamidian H, Soltanian-Zadeh H, Faraji-Dana R, et al Estimating Brain Deformation During Surgery Using Finite Element Method: Optimization and Comparison of Two LinearModels. Springer Science 2008; 55: 157-67.

[4] Hogea CS, Davatzikos C, Birosb G. Brain-Tumor Interaction Biophysical Models for Medical Image Registration. 2008. doi./10.1137/07069208X

[5] Clatz O, Bondiau PY, Delingette H, et al. Brain Tumor Growth Simulation. IRNA; 2004. Report No.: 5187.

[6] Hogea CS, Birosb G, Davatzikos C. Fast Solvers for Soft Tissue Simulation with Application to Construction of Brain Tumor Atlases urll ww.seas.upenn.edu/ biros/papers/brain06.pdf 1. 2007.

[7] Mohamed A, Zacharaki EI, Shen D, et al. Deformable registration of brain tumor images via a statistical model of tumor-induced deformation. Medical Image Analysis 2006; 10: $752-63$.

[8] Clatz O, Sermesant M, Bondiau PY, et al. Realistic Simulation of the 3D Growth of Brain Tumors in MR Images Coupling Diffusion with Biomechanical Deformation. IEEE Trans Med Imaging 2005; 24: 1334-46.

[9] Zacharaki EI, Hogea CS, Shen D, et al. Non-diffeomorphic registration of brain tumor images by simulating tissue loss and tumor growth. NeuroImage 2009; 46: 762-74.

[10] Powathil G, Kohandel M, Sivaloganathan S, et al. Mathematical modeling of brain tumors: effects of radiotherapy and chemotherapy. Physics in medicine and biology 2007;52: 3291-306.

[11] Bach Cuadra M, De Craeneb M, Duaya V, et al. Dense deformation field estimation for atlas-based segmentation of pathological MR brain images. computer methods and programs in biomedicine 2006; 84: 66-75.

[12] Park BJ, Kim HU, Sade B, et al. Meningiomas Diagnosis, Treatment and Outcome. Doi: 10.1007/978-1-84628-784-8 Springer2008.31-65

[13] Kaus M, Warfeld SK, Nabavi A, et al. Automated Segmentation of MRI of Brain Tumors. Radiology 2001; 218(2): 586-91.

[14] Warfeld SK, Ferrant M, Gallez $X$, et al. Real-Time Biomechanical Simulationof Volumetric Brain Deformation for Image Guided Neurosurgery. IEEE transactions on Medical Imaging 2000 0-7803-9802-5.

[15] Ferrant M, Nabavi A, Macq B, et al. Registration of 3-D intraoperative MR images of the brain using a finite element biomechanical model. IEEE transactions on Medical Imaging 2001; 20: 1384-97.

[16] Miller K, Taylor Z, Wittek A. Mathematical models of brain deformation behaviour for computer-integrated neurosurgery. Research Report \# ISML/01/2006

[17] Wittek A, Miller K, Kikinis R, et al. Patient-specific model of brain deformation: Application tomedical image registration. elsevier Journal of Biomechanics. doi:10.1016/j.jbiomech.2006.02.021.

[18] Yousefi H. Ahmadian A, Saberi H, et al. "Brain tumor modeling: glioma growth and interaction with chemotherapy",Proc. SPIE 8285, International Conference on Graphic and Image Processing (ICGIP 2011), 82851M doi:10.1117/12.913432

[19] Wasserman R, Acharya R. A patient-specificthe in vivo tumor model.Mathematical Biosciences1996; 136(2): 11140.

[20] Khodadad D; Ahmadian A; Ay M; et al."B-spline based free form deformation thoracic non-rigid registration of CT and PET images", Proc. SPIE 8285, International Conference on Graphic and Image Processing (ICGIP 2011), 82851K doi:10.1117/12.913422

[21] www.brainweb.com 2011.

[22] www.3Dslicer.org. 2011. 